Die Ankunft des Messias ist ebenso wie die Apokalypse problematisch geworden. Magris lehnt das Versprechen der Erlösung oder der Entelechie von Kultur ab und schreibt den Begriff von Apokalypse um: "Apocalissi, in greco, significa rivelazione, scoprire e rendere manifeste le cose nascoste.$^{58}$ Parusie, Revelation, ist nur noch bezogen auf die Schrecken der Geschichte. Das Gedächtnis hat also die Aufgabe, das Unvergessliche vor dem Vergessen zu schützen, Bildkonstellationen zu suchen, an deren Rändern das Unsagbare aufscheint.

Diese Wanderung des Engels der Geschichte durch verschiedene Kulturen zeigt etwas, das für das Thema dieses Bandes Katastrophen und Gedäcbtnis eminent relevant ist: Der Bindestrich zwischen Gedächtnis und Katastrophe ist nicht snatürlich،. Ihre Verbindung impliziert eine Politik des Gedächtnisses, die das verbindet, was von einet unhintergehbaren Differenz getrennt ist. Die verschiedenen Modelle, die wir skizziert haben, konvergieren trotz unterschiedlicher Wege, Kontexte und Positionierungen zu einem Moment, das uns vielleicht hoffen lässt: Es könnte sein, dass der Engel von Benjamin im 21. Jahrhundert nicht mehr zwischen einer vergangenen Serie von Katastrophen und einer utopischen Zukunft zerrissen ist. Dieser Engel hat einen eigenen Körper, hört seinen eigenen Rhythmen zu, ist vielleicht facettenreich fähig, in den Katastrophen der Vergangenheit die Krisen und Chancen der Gegenwart zu erkennen. Die Politik des Ästhetischen verlangt dem Subjekt gewiss Trauer, Leiden und Exzentrik ab. Aber erst die kritischen Epiphanien einer derartigen Kunst des Gedächtnisses befähigen vielleicht die Kultur zur Potenz, in der Gegenwart Entscheidungswege zu erkennen, die Widerstand und Öffnungen im Geschlossenen möglich machen.
Alexander Honold (Basel)

\section{Im Nervenzentrum der Katastrophe \\ Die Großstadt als traumatischer Gedächtnisraum in Döblins Berlin Alexanderplatz.}

»Die Dinge in diesem Buch Berlin-Alexanderplatz vom Schicksal Franz Biberkopfs sind richtig, und man wird sie zweimal und dreimal lesen und sich einprägen, sie haben ihre Wahrheit, die zum Greifen ist. « (414) ${ }^{1}$ Worin aber besteht diese Wahrheit Franz Biberkopfs, auf die uns die auktoriale Geste des Erzählers zu Beginn des Neunten Buches so vehement hinweist? Sie scheint etwas zu sein, das erst nach wiederholter Lektüre ans Licht tritt, obwohl es doch zum Greifen nahe ist - das fordert den hermeneutischen Zirkel heraus. Etwas, das sich beim Lesen einprägt, zweimal, dreimal, und einen tiefen Abdruck, eine ebenso offenbare wie geheime Spur hinterlässt - das wiederum ruft die Analyse auf den Plan. Und doch muss das Deuten und Analysieren nicht auf eine seigentlicher Bedeutung hinter oder unter dem Roman spekulieren. Denn die gesuchte Wahrheit, so verstehe ich Döblins philologische Ermunterung zum mehrmaligen Lesen, Runde um Runde über den Alexanderplatz, diese Wahrheit ist in die ästhetische Faktur des Textes eingebettet. Sie liegt, wenn wir diesem Hinweis Glauben schenken, nicht zuletzt in der Repetition, in den vielerlei Vorgängen des Wiederholens und Durcharbeitens, denen sich nicht nur die Hauptfigur zu unterziehen hat, sondern auch der oder die Lesende.

Die Geschichte selbst steckt voller Zitate und Wiederholungen. Manche historischen Ereignisse, so hat Marx in seiner Schrift über den 18. Brumaire des Louis Bonaparte eine Bemerkung Hegels aufgenommen, spielen sich zweimal ab, einmal als Tragödie, und ein zweites Mal als Farce. ${ }^{2}$ In Döblins Wallenstein ist zu lernen: Die Geschichte setzt ein, nachdem die Schlachten geschlagen sind. Denn dann beginnt ihre Wiederholung. "Nachdem die Böh-

1 Textgrundlage: Alfred Döblin, Berlin Alexanderplat\%: Kommentierte Gesamtausgabe, Werner Stauffacher (Hrsg.), München 2001; Zitate werden fortlaufend im Haupttext nachgewiesen.

${ }^{2}$ Karl Marx, „Der achtzehnte Brumaire des Louis Bonaparte (1852)«, in: Marx Engels Werke, Bd. 8, Internationale Marx Engels Stiftung (Hrsg.), Berlin 1978, S. 111-207, hier S. 115.

58 Ebd., S. 17. 
men besiegt waren, war niemand darüber so froh wie der Kaiser. $\aleph^{3}$ Dieser erste Satz, ein Nukleus der Döblin'schen Wallenstein-Phantasie, ${ }^{4}$ bricht in seiner dezidierten Nachträglichkeit mit den Konventionen des Historismus. Er nähert sich der Tragödie aus der Perspektive ihrer Wiederholung als Farce. Döblin schlägt einen Erzählton an, der den Wiederholungs-, ja den Zitatcharakter der Geschichte von Beginn an als Index mit sich führt.

Nach dem Krieg auf den Schlachtfeldern beginnt der in den Köpfen. Ein vierjähriger Ausnahmezustand ist zu Ende, der den Helden fernab der zivilisierten Welt festgehalten hatte, im Kasernenton unter Männern einer abstumpfenden Monotonie ausgesetzt, deren Nachhall er nun mit sich herumträgt: »In ihm schrie es entsetzt: Achtung, Achtung, es geht los» (15) Dabei sollte es doch nun eigentlich nicht losgehen, sondern zu Ende sein. »Die Geschichte vom Franz Biberkopf« beginnt, nachdem er seine Haftzeit in Tegel abgesessen hat und in die Stadt zurückkehren kann: "[D]ie vier Jahre waren um《. Es folgt allerdings ein Satz, der die Zeitverhältnisse auf den Kopf stellt und am Anfang eines mehr als vierhundertseitigen Romans von bemerkenswerter Selbstironie seitens des diensthabenden Schriftstellers zeugt: »Die Strafe beginnt. Die Strafe, ihrerseits als juristische Replik das Echo eines Verbrechens, sollte dem Delinquenten im Gefängnis ausgiebig Gelegenheit zum reuevollen Durcharbeiten seiner Tat bieten, deren Wiederholung allerdings gerade vereiteln. Nun steckt freilich die Geschichte Franz Biberkopfs, das verrät schon ihr Anfang, voller Wiederholungen. Auf das Absitzen der Gefängnisstrafe folgt ihre Wiederkehr als Farce, die sogenannte Freiheit. Doch bleibt Biberkopfs Weg in die Freiheit, auf dem der Held mit aller Gewalt von den Tentakeln der Großstadt eingezogen wird, geradezu starrsinnig fixiert auf das, was er hinter sich hat: »Er drehte den Kopf zurück nach der roten Mauer, aber die Elektrische sauste mit ihm auf den Schienen weg, dann stand nur noch sein Kopf in der Richtung des Gefängnisses.« (15)

3 Alfred Döblin, Wallenstein, Walter Muschg (Hrsg.), München 1983, S.9.

4herosch gesehen handelt es sich um eine bysteron-proteron-Struktur, denn das in Rheton nachträglich nachzuvollziehende Vorzeitigkeit Geschehene wird auf eine erst nehtanglich noch Weise vorausgesetzt. Dazu Axel Hecker: wWeder die Bohmen noch der Kaiser sind durch das Vorstehende (Titel und Inhaltsangabe) eingeführt; der bestimmte Artikel scheint an einen vorausgehenden Kontext anzuschließen, der de facto Artiel Eröffnung nicht besteht. Obendrein ist die Stellung mehr als ungewöh eines Satzes durch einen temporalen Nebensatz mit snachdem ist nur dann üblich, wenn diese Beziehung explizit sthematisierendk ist, das heilst ein durch den Konwent dann, mit dem betreffenden Satz, abgerufenes Ereignis in-

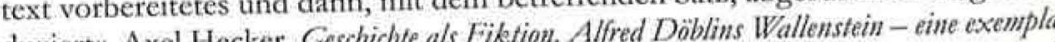

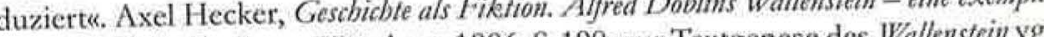
riscbe Kritik des Realismus, Würzburg 1986, S. 190; zur Textgenese des Wallenstein vg ebd.
Über kurz oder lang werden andere Anstalten sich den Entlassenen holen. Unter solchem ,Wiederholungszwang scheinen Haft- und Heilanstalten besonders stark zu leiden. Und erst recht Franz Biberkopf selbst. Die Wiederholung ist in seiner Geschichte omnipräsent und als Textphänomen selbst bei erstmaliger Lektüre unübersehbar. Was auf den ersten Seiten steht: die roten Mauern von Tegel, die Dächer und blanken Scheiben, die von den Häusern zu stürzen scheinen - das bleibt eben dort nicht stehen. An den Traufkanten der Berliner Mietskasernen halten sie zwar, diese Dächer, doch rutschen sie stattdessen scheinbar unkontrolliert durch die erzählte Geschichte. In einem Straßenbahnwagen der Linie 41, vom Tegeler Gefängnis bis zum Rosenthaler Platz, fährt Biberkopf durch die Stadt, die Stadt aber fährt mitten durch ihn:

Die Wagen tobten und klingelten weiter, es rann Häuserfront neben Häuserfron ohne Aufhören hin. Und Dächer waren auf den Häusern, die schwebten auf den Häusern, seine Augen irrten nach oben: wenn die Dächer nur nicht abrutschten, aber die Häuser standen grade. (17)

An Rilkes Malte Laurids Brigge ist bei solchen Passagen zu denken, dem die Straßenbahnen durch Hirn und Stube jagen, an das Weltende-Gedicht Jakob van Hoddis', an Ludwig Meidner und andere, auf deren Großstadtbildern über kleinen, verängstigten Menschenleibern zerfließende Straßenschluchten zusammenschlagen.

$\mathrm{Ob}$ der Beobachter stillhält und die Stadtkulisse an ihm vorbeirauscht oder umgekehrt, ist kaum zu unterscheiden. Thr Verhältnis ist nicht kontemplativer Art, sondern eines der Resonanz; ein Abgeben und Aufnehmen von Schwingungen, von überfallartigen akustischen Innervationen, deren Gewalt mit dem Begriff »Lärm« nur unzureichend beschrieben ist. In Biberkopf drinnen hallt es, da dröhnen tumultuarische Klangprozessionen von Befehlen, Ausrufen, Sinnsprüchen, unterlegt mit der obstinaten Rhythmik militärischer Marschmusik. Dieses Dröhnen muss aus ihm heraus, muss der Stadt zurückgegeben werden, da kommen ihm die engen dunklen Hinterhöfe der Sophienstraße gerade recht. In sie flüchtet Biberkopf vor dem Straßenlärm, um seine eigene Lärmschlacht zu schlagen: „Und plötzlich sang er schallend los, sang die Wände an.« Was in Biberkopf tönt und klingt, das wird umgestülpt, nach außen gekehrt, bis es die ihn peinigenden Häuserfronten zum Erzittern bringt:

Von den Wänden kam der Ton wieder. Das war gut. Seine Stimme erfüllte seine Ohren. Er sang mit so lauter Stimme, wie er im Gefängnis nie hätte singen dürfen. Und was er sang, daß es von den Wänden widertönte? »Es braust ein Ruf wie Donnerhall.« Kriegerisch fest und markig. Und dann: »uvivallerallera« mitten aus einem Lied. (18) 
Sein Singen ist »schallend«, was keineswegs tautologisch zu verstehen ist, sondern datauf abhebt, dass der Zweck unmittelbarer physischer Affektion durch die Druckwellen intentional mit dem gewählten musikalisch-poetischen Programm verbunden ist. Es geht um Bewegung, um Erschütterung von Mensch, Haus und Hof durch die schiere akustische Wucht des Biberkopf-Gesangs. Nicht von ungefähr bedient er sich patriotischen Liedgutes, ${ }^{5}$ das im zitierten Vers einen Ruf wie Donnerhall brausen lässt, d. h. auf meta referentieller Ebene die eigene akustische Rezeptionswirkung thematisiert. Marschieren und kräftig Ausschreiten lässt es sich zur Wacht am Rhein obendrein, ebenso zu dem geschmetterten Juvivallerallera-Refrain von Schwarzbraun ist die Haselnuss. Die auch im Falle Biberkopfs betonte »kriegerische« Bedeutung seiner gegen die Hauswände gerichteten Sangeskraft erinnert an die berühmte biblische Geschichte der Posaunen von Jericho. Dieses früheste schriftlich verbürgte Straßenkonzert war der belagerten Stadt in der militärischen Absicht dargeboten worden, ihre Befestigungsmauern durch den Schalldruck der eingesetzten Blechblasinstrumente zum Einsturz zu bringen. Manchmal, so belegt dieses Beispiel, sind Sang und Klang von einer Vehemenz, deren man sich nur schwer zu erwehren vermag. ${ }^{6}$

Biberkopfs Singen trifft naturgemäß nicht auf ungeteilte Zustimmung: Laß ihn schrein, soviel er will. Laß ihn tun und machen. Aber nicht bei mir.« (20) Biberkopf singt nicht freiwillig. Wie ein gutes Jahr später sein tage- und nächtelanger Schreikrampf im Festen Haus der Psychiatrischen Klinik von Berlin-Buch, so hat auch das Singen im Hinterhof eine sowohl traumatische wie therapeutische Dimension. Er singt, weil es im Gefängnis hieß: "Alles Singen, Pfeifen, Lärmen ist verboten.« (19) Zugleich aber singt er, auf eine paradoxe Weise, auch vom Gefängnis:

5 Zur Entschlüsselung der angespielten Lieder - Volks- und Marschlieder, aber Zur En zeitgenössischer Unterhaltungsmusik - haben auch populärer Operetten und zeitgenössischer Unteraltungäusk - haben Werner Stauffachers Werkkommentar und Gabriele Sanders Erlauterungen (Gabriele Sander (Hrsg.), Alfred Döblin. Berlin Alexanderplatz. Erläuteringen und Dokumente, Stuttgart 1998) eine Fülle von wichtigen Hinweisen gegeben; allerdings finmenter Schuarzbatn ist di det sich der zum w) uvivallerallerak-Refrain gehorende

Haselnuss in beiden Kom Hinterhof hat Döblin in seinem Berlin-Feuilleton Östlich sum dexauderplatz des Jahres 1923 zuerst geschildert. Sie ist eine motivische Iie Müller-Salget in seiner Studie zur EntstehungsmInitialztindangm des Roul geschichte herausgearbeitet hat (Klaus Müller-Salget, "Zur Entstehung von Berlin Alexanderplatæw, in: Matthias Prangel (Hrsg), Materialien z" Alfred Döblin „Berlin Alexanderplatz, Frankfurt a.M. 1975, S. 117-135, hier S. 121).
Der Entlassene saß allein. Es braust ein Ruf wie Donnerhall, wie Schwertgeklirr und Wogenprall. Er fuhr mit der Elektrischen, blickte seitlich hinaus, die roten Mauern waren sichtbar zwischen den Bäumen [...]. Die Mauern standen vor seinen Augen, sie betrachtete er auf dem Sofa, betrachtete sie unentwegt. Es ist ein großes Glück, in diesen Mauern zu wohnen, man weiß, wie der Tag anfängt und wie er weiter geht. (19)

Weil die roten Mauern nicht aufhören wollen, sondern immer wieder erscheinen, und ihr disziplinär geregelter Tageslauf so viel mehr Halt gegeben hat als die Freiheit und die hohen Dächer der Großstadt - deshalb muss Biberkopf singen. Seine Lieder hat man ihm einst in einem vierjährigen Ausnahmezustand eingebläut, dessen Wirkungen tiefer sitzen als die vier Jahre Tegel.

Es sind »Kriegslieder« (20), die Biberkopf singt. Weil sich aber die eingängigen Liedzeilen aus der Wacht am Rhein (1870) oder vom Uhlandschen Guten Kameraden 7 aus dem Gedächtnis nicht verdrängen lassen, so oft Biberkopf auch versucht, sie hinauszusingen, durchziehen ihre Auftritte den Roman nicht minder penetrant als die Erinnerungen an T'egel. Später kommt noch die Weise vom Scbnitter Tod hinzu und anderes Zitatengut. »Leitmotive«, so könnte man ihre behartliche Wiederkehr auf den Begriff bringen, wenn es sich um ein Werk aus der Feder des bekennenden Wagnerianers Thomas Mann handeln würde. Aber in Berlin Alexanderplatz geht es nicht um die Kunst der Charakterzeichnung und ihre mehr oder minder subtilen dramaturgischen Anschubwirkungen; und auch nicht darum, einem unüberschaubaren Gewirr von Nebenfiguren für ihre weitgestreuten Auftritte jeweils ein Glöckchen umzuhängen, an dem sie dann wiederzuerkennen sind. Die Wiederholungseffekte in Döblins Roman sind von gänzlich anderer Art. Sie sind nicht beiherlaufende ästhetische Signale, welche als Lesehilfen die eigentliche Handlung kommentieren und vereinfachen. Nein, die Wiederholungen sind ein Teil des Geschehens selbst, sie strahlen auf die Figuren des Romans zurück, werden von ihnen selbst erlitten oder gar verursacht.

Das Gesetz der Wiederholung ist es, das den entlassenen Biberkopf in die Ackerstraße treibt, zur Schwester jener Ida, die er vor Jahren totschlug im Affekt. Und wieder braucht er Gewalt: »Minna kann ihre Hand nicht loskrie-

7 Das am meisten gesungene Soldatenlied der ersten Kriegswochen bestand aus Dichtung und Volkes Stimme gleichermaßen. Den Beginn bildeten die ersten Zeilen aus Der gute Kamerad von Ludwig Uhland (1809 entstanden); aus dem Geist der Stunde dagegen wurde der Refrain hinzugesetzt, der in der zweiten und dritten Strophe gleich lautet: »Ich hatt' einen Kameraden, / Einen bessern find'st du nit, / Die Trommel schlug zum Streite, / Er ging an meiner Seite. Gloria, Gloria, Gloria, Viktoria! / Mit Herz und Hand fürs Vaterland, fürs Vaterland! 
gen, [...] da kann man nichts machen, solche Männerarme sind aus Eisen, Eisen. Ich schrei Hilfe. Sie schrie. [...] Und - sie hat richtig gesehen. Jetz weiß sie, sie ist die Schwester von Ida, so hat er manchmal Ida angeschaut. (39) Das nämliche Prinzip waltet in Reinholds nach gleichbleibendem Muster ablaufenden Frauengeschichten, und sogar noch in seinen schließlichen Durchbrechungen dieses Stereotyps. Mit Cilly, die er wie andere abgelegte Geliebte vor ihr an Biberkopf weitergeschoben hatte, plant Reinhold plötzlich eine »Reptise«. Bei Mieze, der neuen und so anhänglichen Freundin Biberkopfs, will Reinhold den Frauentausch in umgekehrte Richtung wiederholen und begeht dabei eine Reprise des von Biberkopf verübten Verbrechens. Dass schließlich Biberkopf wiederholt zu Reinhold zurückkehrt, der ihn erst um seinen rechten Arm, dann um die Geliebte brachte, ist nicht allein treuherziger Naivität geschuldet, sondern ebenfalls dem Prinzip, dass sich Täter wie Opfer alles zwei- und dreifach sagen lassen müssen. Und immer wieder durch dieselben Straßen laufen, an denselben Ecken stehen, in den gleichen Kneipen hängenbleiben. Die ganze Stadt ist nichts anderes als eine unendliche Wiederholung und Kopic ihrer selbst. Trotz seiner Größe ist in diesem Berlin jeder fast jederzeit wiederzufinden.

Und Franz fängt an, die Stadt zu betrachten wie ein Hund, der eine Fußspur verloren hat. Was ist das für eine Stadt, welche riesengroße Stadt, und welches Leben, welches Leben hat er schon in ihr geführt.« (387) Hier hat man keinen Lebenslauf, sondern eine topographische Legende; mit festen Markierungen im Stadtplan an jenen Plätzen und Punkten, die Zeugen früherer Abschnitte der Handlung waren: »Am Stettiner Bahnhof steigt er aus, dann zieht er die Invalidenstraße lang, da ist das Rosenthaler Tor. Fabisch Konfektion, da hab ick gestanden, ausgerufen, Schlipshalter vorige Weihnachten. Nach Tegel fährt er mit der 41.« (387) Franzens Fahrt nach Tegel nach dem Tod von Mieze ist schon eine Wiederholung der Wiederholung Ein früheres Mal bereits war er mit der 41 nach Tegel hinaus-, oder besser gesagt: zurückgefahren, wie zur Selbstvergewisserung. Nach allem, was ihm inzwischen in der Freiheit widerfahren war, den Schicksalsschlägen durch Lüders und Reinhold, zieht die Wiederbegegnung mit Tegel eine Art Bilanz:

Und Franz Underter. Ja das and steht vor Fabisch an der Haltestelle, will er! Er steht da und wartet und fil Nach Tegel, Gefängnis, Gefängnismauer! Da will er hin. Da muß er hin. Und dann geschieht es, daß die 41 kommt, hält, und Franz steigt ein. Er fühlt, das ist richtig. geschieht Elektrische fährt ihn nach Tegel. [...] Brunnenstraße, Abfahrt, und fahrt, und die Elekter Uferstraße, Alleen, Reinickendorf, es ist wahr, das gibt es alles, da fährt er hinein, es steht da. (283)
Sein höchstpersönlicher Stadtplan von Berlin ist genordet, ist auf Tegel geeicht. Auch wenn dort nur der schon hinlänglich bekannte Anblick der roten Mauern auf ihn wartet, hat die Wiederbegegnung einen Wert. Immerhin sind es Außenmauern, die er abfährt in seiner Straßenbahn; immer noch ist er frei:

Und wie die roten Mauern auftauchen, links die roten Mauern, die schweren Eisentore, ist Franz stiller. Das ist von meinem Leben, das muß ich betrachten, be trachten. Die Mauern stehen rot, und die Allee zieht davor lang, die 41 fährt dran vorbei, General-Pape-Straße. West-Reinickendorf, Tegel, Borsig hämmert. (387)

Die wiederholte Rückkehr zur Strafanstalt, man könnte sie im Sinne einer erfolgreichen Resozialisierung als intensive Auseinandersetzung mit dem begangenen Unrecht deuten, und tatsächlich fällt Biberkopfs Blick dort auf das versammelte Böse:

An allen Fenstern stehen Gefangene, stoßen die Köpfe gegen die Stangen [...]. Da stehn Mörder, Einbruch, Diebstahl, Fälschung, Notzucht, die ganzen Paragraphen, und klagen mit grauen Gesichtern, da sitzen sie, die Grauen, jetzt haben sie Miezen den Hals eingedrückt. (388)

Weil hier das geballte juristische Regelwerk in tristen Verbrechervisagen Spalier steht, ist das Tegeler Gefängnis im Gefüge der Großstadt ein exterritorialer Raum. Es ist ein Ort der Verbringung, Verwahrung und Verdrängung und gleicht darin anderen Disziplinarmächten wie der Schule, dem Militär oder den psychiatrischen Kliniken. Je unüberwindlicher und unbeugsamer sie sich gerieren, umso länger tragen die Menschen diese Institutionen mi sich herum: »Ich - träume von der Schule wie ein anderer nach einem Unfall!k vertraute Döblin in einem autobiographischen Abriss von 1928 seinen Lesern an. ${ }^{8}$

Und aus genau diesem Grunde melden sich auch Tegels rote Mauern immer wieder zu Wort. Nicht nur als Schauplatz oder Motiv, sondern öfter noch als Chiffre eines unbestimmten Leidens, das auf ein traumatisches Erlebnis zurückgeht und dessen Auftreten mit kleinen, unkontrollierbaren Flashbacks verbunden ist: „Das Zittern läuft über ihn, ohne daß ers will Er ist wo beim Alex auf dem Einbruch, es ist alles weg von ihm, das muß mit dem Unfall zusammenhängen, das sind die Nerven.« (296) Der Unfall jedoch, bei dem Biberkopf vom Auto überrollt wurde und anschließend seinen rechten Arm verlor, ist nicht Auslöser, sondern symptomaler Bestandteil in einer Verkettung und Verschiebung von Wiederholungsfiguren. Biber-

8 Alfred Döblin, "Erster Rückblick«, in: ders., Im Buch - Zu Haus - Auf der Straße, vorgestellt von Alfred Döblin und Oskar Loerke (1928), mit einer Nachbemerkung von Jochen Meyer, Marbach 1998, S.7-126, hier S. 78. 
kopfs Neigung, dasselbe nochmals zu tun unter anderen Vorzeichen, folgt widerstreitenden Impulsen; es ist der Versuch, eine schmerzhafte Vergangenheit $\mathrm{zu}$ berühren, ohne ihr wirklich nahekommen zu müssen. In seinem psychiatrischen Gutachten wird ihm attestiert: »Alles wehrt sich in dem Mann, diesen Weg zurückzugehen. Der ist wie versperrt. [...] Es war ein psychisches Trauma.« (445)

Den Weg zurückzugehen, mit der Linie 41 retour, das bedeutet - wenn wir die Kriegsgesänge Biberkopfs ernst nehmen - eine Lektüre in Gegenrichtung: alles zurück auf die 14, zum Augusterlebnis der Mobilmachung und den vier Jahren danach. Biberkopfs Trauma ist, so haben Wolfgang Schäffner und Armin Schäfer festgestellt, nichts anderes als eine Kriegsneurose. ${ }^{9}$ In drei großen Romanen, so Schäffner, habe Döblin sein psychiatrisches Wissen dazu genutzt, Protagonisten mit traumatischen Kriegsschäden darzustellen:

. [...] den Kriegsneurotiker Grabenkrieg bei Arras an Anratselitterverletzung regelmäßig in Delirien verNovember 1918 nach einer Granatssplitterverletzung regelmaßig in Delirien verfällt; Edward Allison, der in Hamlet oder Die lange Nacbt nimmt ein Ende (1946) einen Kamikaze-Angriff im Zweiten Weltkrieg mit hartnäckigen kriegstraumatischen Symptomen überlebt. ${ }^{10}$

Auch kleinere Prosaarbeiten wie die Ermordung einer Butterblume oder Die beiden Fresndinnen und ibr Giffmord bringen Wahtnehmungs- und Handlungsmuster zur Anschauung, die als lehrreiche Störfälle des psychiatrischen und juristischen Normalisierungsdrucks angelegt sind. Anders als empirische Personen, die sich durch Kategorien wie diejenige der verminderten Zurechnungsfähigkeit jederzeit einordnen lassen müssen, können literarische Figuren im Eigensinn ihres bizarren Erlebens verharren - und von diesem Privileg machen sie bei Döblin reichlichen Gebrauch. Auch an Robert Musils Frauenmörder Moosbrugger ist in diesem Kontext zu erinnern, wie Biberkopf ein Geschöpf der zwanziger Jahre, in dessen Krankenakte sich die verschiedensten und widersprüchlichsten Definitionen psychosozialer Devianz ein Stelldichein geben und um Moosbruggers Seele spielen. ${ }^{11}$

9 Armin Schäfer erwähnt in seiner Untersuchung über die Anthropologie Hann Armin Schafer en Henny Jahnns beilafig auch Franz Biberkopf, wesäk Kühren kanne (Armin SchäGastrauma und eine Verschüttung im Graben zuruckfuhren kan (Armin Schafer, Biopolitite des Wissens. Hanns Henny Jabnns literarisches Archiv des Menschen, Würzburg 1996, S. 296, Anm. 114.)

10 Wolfgang Schäffner, Die Ordnung des Wabns. Zur Poetologie psychiatrischen Wissens bei Alfred Döblin, München 1995, S. 360 f

11 Vgl. Alexander Honold, Die Stadt und der Krieg. Raum und Zeitleonstruktion in Robert Musils Roman or Mann obue Eigenscbaften«, München 1995.
Immer wieder reflektiert Döblin an exemplarischen Fallgeschichten und Modellstudien die dramatischen Veränderungen auf dem Feld der klinischen und forensischen Psychiatrie. Vor 1900 hatte sich die psychologische Forschung vorrangig mit Phänomenen der Hysterie, die psychiatrische dagegen hauptsächlich mit Paranoia beschäftigt, aus deren höchst verschiedenartigem Formenkreis Kraepelin und Bleuler alsbald die neuen Ätiologien der Dementia praecox und der Schizophrenie ausgliederten. ${ }^{12}$ Döblin, der in Regensburg und Berlin mehrere Jahre in psychiatrischen Kliniken gearbeitet hatte, war mit dem Stand der Debatte bestens vertraut. In Berlin-Buch konnte er zwischen 1906 und 1908 auf einer Beobachtungsstation für Kriminelle Krankheitsformen und Behandlungsmethoden studieren, die dann als literarisches Material wiederkehren. Später waren es vor allem die sogenannten Materialschlachten an der Westfront, die Nervenärzte mit massenhaften pathologischen Befunden wie Tremor, Lähmungserscheinungen, Sprach- und Wahrnehmungsstörungen versorgten. Kriegsbedingte Neurosen, so manifest sie waren, wurden keineswegs von allen Psychiatern als Krankheitsgrund akzeptiert, im Gegenteil. ${ }^{13}$ Die nervlichen Schädigungen wurden, statt auf starke mechanische und psychische Erschütterungen etwa durch Granatexplosionen, vorzugsweise auf Fehlbildungen oder Fehlverhalten der Soldaten selbst zurückgeführt - und entsprechend forsch behandelt. Da nicht etwa Heilung, sondern die erneute Kriegsverwendungsfähigkeit oberstes Ziel war, kam es weniger auf Ursachenforschung an als darauf, die Therapie für den Patienten nicht angenehmer erscheinen zu lassen als den Fronteinsatz. Tatsächlich führten die verabreichten Elektroschocks, Hypno-

12 In der 1893 erschienenen vierten Auflage von Kraepelins Lehrbuch Psychiatrie wird erstmals der Begriff der »Dementia praecox« eingeführt (vgl. Schäffner, Die Ordnung des Wahns, S. 61), der Begriff »Schizophrenie« bei Bleuler um 1908. »Die neuen psychiatrischen Verfahren und Praxen formen das ganze Material um: Die Klassifikationsgruppen ,Dementia praecox bzw. 'Schizophrenier übernehmen nahezu vollständig die große Anzahl der Paranoiker, die noch in den $80 \mathrm{cr}$ Jahren die Anstalten bevölkerten.« (Ebd., S. 351).

13 Erst bei der Kriegstagung der deutschen Psychiater 1916 in München wurde die psychogene Natur der Neurosen mehrheitlich bejaht; denn »unter dem Druck massenhafter Kriegsneurosen und dem Erfolg der hypnotischen Gewalttherapien« versprach allein diese Erklärung, die krankmachenden Verhältnisse selbst unangetastet zu lassen. Dagegen hatte Hermann Oppenheims Erschütterungstheorie des Traumas die Funktionsstörungen im zentralen Nervensystem durch ein Zusammenwirken psychischer mit mechanischen Faktoren erklärt. Dadurch wäre es möglich gewesen, »einen direkten Zusammenhang zwischen Granatexplosion, Schock und neurotischen Symptomen herzustellen, wie es das maschinale Dispositiv des Schocks nahelegt.« (Schäffner, Die Ordnung des Wabns, S. 366f.). 
sen, Scheinoperationen oder quälendes Zwangsexerzieren dazu, dass die solcherart Behandelten lieber in die Lebensgefahr der Schützengräben zurückflüchteten. ${ }^{14}$ Während der Krieg mit seinen technophysischen Effekten in der Ätiologie der Neurosen zunehmend verdrängt wurde, zog er in die Therapie selbst ein; die Behandlungsmethoden, so nochmals Schäffner, wurden »dem Geschehen auf den Schlachtfeldern immer ähnlicher. « ${ }^{15}$

Um 1920, also im psychopathologischen Nachfeld des Krieges, beschäftigen sich die Arbeiten Sigmund Freuds (Das Unbeimliche, Jenseits des Lustprinzips) mit den Manifestationen von Wiederholung und Zwang, mit Stereotypen und Automatismen. Die starre Wiederholung fixierter Handlungsmuster oder die zwanghafte Wiederkehr halluzinierter Sinneseindrücke, so erkannte er haben oft einen traumatischen Erlebniskern zum Inhalt, dessen pathogene Energien durch das beständige Umkreisen und Aufrufen zum Abklingen gebracht werden sollen. Im Zusammenhang damit entwickelte Freud eine Theorie des Schocks als plötzlicher und gewaltsamer Durchbrechung des vom Bewusstsein errichteten Reizschutzes. ${ }^{16}$ Psychischer oder habitueller Wiederholungszwang können helfen, diesen Schutzschild wiederaufzubauen; einen vergleichbaren Trainingseffekt, so ergänzte Walter Benjamin, haben die gesteigerten Wahrnehmungsintensitäten der Großstadt oder des Kinos. ${ }^{17}$ Dass es vor allem die im Weltkrieg erlittenen, schockhaften Erlebnisse waren, die zu schwersten psychischen Schädigungen führten und von ihren Patienten imaginär immer wieder durchlaufen und wiederholt werden mussten, hat Döblin in seinem bereits erwähnten autobiographischen Essay von 1928 ausführlich dargelegt:

Im Krieg sind viele erkrankt nach Erschütterungen, Granatexplosionen, Bombenabwürfen. In ihren Träumen trat immer diese Situation vor sie; beängstigte sie. Warum? Es sind keine Mörder. Die Leute sucht im Traum wieder die Situation heim, die sie überrascht hat. Das ist die Gegenreaktion ihrer Seele. Sie ist erkrankt, hich im Traum die weil sic sich damals nicht wh Situation vor, geht sie von neuem an, und allmählich erstarkt sie daran. Der

14 „Den massenhaften psychischen Störungen der Soldaten wie Tremor, Lähmungen, A Aphonie, Sensibiliars und Schstorrengen benkrieg an der Westfront versuchten die Psychiater mit verschiedensten Gegenmaßnahmen zu begegnen: Hypnose, Scheinoperationen, Ströme.« (Ebd., S. 370)

15 Ebd., S. 378.

I6 Sigmund Freud, Jenseits des Lustprinzips. Studienausgabe, Bd. 3, Alexander Mitscherlich (Hrsg.), Frankfurt a.M. 1982, S. 241.

17 Walter Benjamin, »Über cinige Motive bei Baudelaire«, in: ders., Gesammelte Schri en, Bd. 1, Rolf Tiedemann/Hermann Schweppenhäuser (Hrsg.), Frankfurt a.M. $1972 / 1989$, S. $605-653$.
Schock heilt aus, das Gleichgewicht zwischen innerer Kraft und äußerem Stoß wird wieder hergestellt. ${ }^{18}$

Döblin selbst attestiert sich einen solchen traumatischen Wiederholungszwang für die familiäre Katastrophe des 1888 nach Amerika getürmten Vaters, ${ }^{19}$ dessen Geschichte in gleich drei divergenten Versionen repetiert wird, ${ }^{20}$ und für die jahrelangen Demütigungen während seiner Berliner Schulzeit. Das hier im autobiographischen Kontext Entwickelte hat Döblin, so meine Vermutung, durchaus im Hinblick auf die gleichzeitige Arbeit an Berlin Alexanderplatz formuliert. Die ihn zeitlebens prägende Erfahrung seiner Ankunft in Berlin nennt Döblin »gewissermaßen eine Nachgeburt ${ }^{21}$ und als symbolischen Vorgang einer wzweiten Geburt« hat man auch die Anfangsszene des Romans verstanden, in der die blutroten Mauern ihren Schützling entlassen und in die Stadt austreiben. ${ }^{22}$ Ein entborgenes Leben, eine Strafe beginnt. Traumatische Fixierungen lässt Biberkopf erkennen, der zwar de iure ein verurteilter Totschläger ist, de facto aber als Kriegsneurotiker in immer neuen Anläufen seine Erlebnisse im Grabenkrieg durchzuarbeiten versucht - und dies bis fast ans Ende des Romans vergeblich. Der Held selbst bringt die Gefängnis- und die Kriegsjahre als die entscheidenden Bruchstellen seines Lebens ausdrücklich in einen Zusammenhang: »Erst bei de Preußen im Graben und dann in Tegel.« (36) Doch erst mit Biberkopfs Zwangstherapierung in der Irrenanstalt Buch lockert sich die Blockade. Ein offenes Wort unter Ärzten:

18 Döblin, »Erster Rückblick«, S. 78 f.

19 "Die verräterische Flucht des Vaters blieb eine nie ganz heilende Wunde, zugleich aber eine nie versiegende Quelle der Phantasie." (Dietmar Voss, Ströme und Steine. Studien zur symbolischen Textur des Werkes von Alfred Döblin, Würzburg 2000, S. 65.) In dieser traumatischen Erfahrung des Vaterverlustes sieht Voss eine mit dem Topos des offenen Meers liierte ambivalente Faszination angelegt, die sich später in der Poetologie des Autors unbewusst Geltung verschafft habe als dominante Symbolik des Strömens, Flutens und Fließens. Trotz der negativen Besetzung des vom Vater zurückgelegten Meeres (das »väterliche« ist das »dämonische Fluidum«) verheißt das Medium des Wassers elementare Geborgenheit; »in Döblins imaginativem Universum [ist] ein Schweben in tragenden, vertrauenerweckenden Strömen die maßgebliche Figur des Glücks« (ebd. S. 68).

20 „Du mußt ran, zum drittenmal. Du sollst noch einmal davon sprechen. - Aber was denn? Von dieser Sache? Ich hab es schon zweimal gesagt. Warum denn? - Du wirst es schon sehen, du weißt es schon, fang nur an.« (Döblin, »Erster Rückblick«, S. 28).

21 Ebd., S. 11.

22 Bernd Widdig, Männerbünde und Massen. Zur Krise männlicher Identität in der Literatur der Moderne, Opladen 1992, S. 160. 
Sehen Sie, Elektrizität ist schon gut, schon besser wie das Gequatsche. Aber nehmen Sie ein ken, dann können Sie was erleben. Kennt man ausm Krieg, Starkstrombehandlung, Mann Gottes. Das ist nicht erlaubt, moderne Folter. (426)

Biberkopf also darf sich mit Recht in die Schreckenswelt von 1914ff. zurückversetzt fühlen: »Und Rollen von Eisenbahnen, Kanonen krachen, Platzen der Handgranaten, Sperrfeuer, Chemin des dames und Langemarck, Lieb Vaterland magst ruhig sein, lieb Vaterland magst ruhig sein. Die Unterstände verschüttet, hingesunken die Soldaten.« (443)

Die schwere Kriegsneurose durch Verschüttung des Grabens nach Granateinschlägen ist in seinem Fall unschwer zu rekognoszieren, da im Text fast schulmäßig durchgeführt. Thr offenbares Geheimnis steht allerdings in merkwürdigem Kontrast zu der geringen Beachtung, die der Erste Weltkrieg als Hintergrund des Romangeschehens bislang erfahren hat. Es ist, als hätte die Logik der Verdrängung die Aufmerksamkeit auf allerlei bedeutsame Begleitumstände des Biberkopfschen Traumas gelenkt: sei es die schwierige Resozialisierung Haftentlassener oder die politische Polarisierung unter dem Druck der Arbeitslosigkeit, die Entwertung des Individuums im Moloch Großstadt oder die Unfähigkeit des Mannes zu gewaltfreien Liebesbeziehungen. Dass es aber für viele Sonderbarkeiten in Biberkopfs Symptomkatalog eine gemeinsame Ursache gibt, scheint dabei ein wenig aus dem Blick geraten.

Dabei war der große Krieg, wie er in Europa allgemein genannt wurde, in der Literatur der späten zwanziger Jahre präsenter denn je. Die »Kriegsbücher«, Frontmemoiren und Romane über den Ersten Weltkrieg sind ein Phänomen der späten Weimarer Republik. Nachdem eine erste Welle nationalistischer Kriegsromane und Erinnerungen bereits abgeebbt war und mit Arnold Zweig (Der Streit um den Sergeanten Grischa) und Ludwig Renn (Krieg) zwei Darstellungen aus dezidiert antimilitaristischer Perspektive vorlagen, erschien im Januar 1929 mit Remarques Im Westen nichts Neues ein weiterer Roman über die Fronterfahrungen, der zum »Weltbestseller in Millionenauflagen ${ }^{23}$ avancierte. Der Kulminationspunkt der literarischen Kriegsthematik lag also, wenn man die Vorabdrucke aus Berlin Alexanderplatz einbezieht, annähernd zeitgleich mit dem öffentlichen Auftritt Franz Biberkopfs. Ins weitere Umfeld gehören die anderen großen Zeitromane der klassischen Moderne, dic auf den Epochenbruch von 1914 fokussiert sind, und zwar meist so, dass sie, wie das paradigmatisch im Zauberberg oder im Mann obne

23 Bärbel Schrader, „Vorbemerkung« zu: dies. (Hrsg.), Der Fall Remarque. »Im Westen nichts Neuese. Eine Dokumentation, Leipzig 1992, S. 5-15, hier S. 5.
Eigenschaften realisiert ist, ${ }^{24}$ sich der Chiffre 1914 in einem Modus des Indirekten nähern. Die feine Tischgesellscháft im Schweizer Sanatorium, der Wiener Hofadel mit seinen auf 1918 terminierten Jubiläumsverlegenheiten: Sie alle befinden sich im Vorlauf zum Kriegsausbruch, dessen »Donnerschlag« ihre Geschichte beenden wird. ${ }^{25}$

In diesen panoramatischen Werken, die sich als Anatomie einer ganzen Gesellschaft verstehen, ist die Anwesenheit des Krieges nicht auf thematischer, sondern auf symptomatologischer Ebene zu suchen. Denn die Beziehung der gesellschaftlichen Lage zum Kriege ist ebenso unabweisbar wie kompliziert, kein Fall für einsträngige Ursachenforschung oder Täterermittlung. Kunstvoll orchestrierte Vorzeichen stigmatisieren die Vorkriegsgesellschaft als moribund, nicht minder vielfältig sind die Folgespuren dieses Epochenbruchs. Die Kultur pflegt, solange sich der Krieg leise grollend in ihrem Epizentrum befindet, zu seiner Nennung und Darstellung ein tangentiales Verhältnis. Kaum irgendwo dürfte man dieses eleganter und zugleich treffsicherer ausgedrückt finden als in Hugo von Hofmannsthals 1917 entstandenem Lustspiel Der Schwierige. Dort ist nicht die schwitzende, zappelnde, derbe Spielart des Kriegsneurotikers zur Besichtigung freigegeben, sondern die subtil neurasthenische Variante. Der Protagonist Hans Karl Bühl ist ein in der misanthropischen Tradition stehender Eigenbrötler und Hagestolz, der sich beharrlich allen Versuchen seiner Verheiratung zu entziehen vermag, bis er am Ende - schließlich sind wir in der Komödie - eben doch unters glückliche Ehejoch tritt. Dieser Herr Karl nun verdankt seine mangelnde Umgänglichkeit, die ihn zum "Schwierigen« stempelt, dem Umstand, etliche Jahre »draußen" gewesen zu sein. Von seinen Kriegserlebnissen ist nie anders die Rede als in der leersten aller Paraphrasen. (Auch bei Döblin fällt mehrfach dies Schlüsselwort anathematischer Fronterfahrungen.) ${ }^{26}$ "Nach allem«, was er »draußen durchgemacht« hat, gilt Hans Karl der stets amüsierbereiten Gesellschaft teils als Spielverderber, teils als Hypochonder. Seine

24 Ähnlich auch Josef Roths Kapuzinergruft und Hermann Hesses Demian; vgl. Honold, Die Stadt und der Krieg.

25 Zur Analogie des Zauberberg-Schlusses mit dem Alexanderplatz vgl. Helmut Koopmann, »Der Schluß des Romans Berlin Alexanderplatz - eine Antwort auf Thomas Manns Zauberberg«, in: Internationale Alfred-Döblin-Kolloquien, Münster/Marbach 1989/1991, S. 179-191, der allerdings ungenau bei Döblin von der Vorahnung eines kommenden Krieges spricht.

${ }^{26}$ So, wenn sich Biberkopf über die Kritik seitens sozialdemokratischer Kneipenkumpane erregt: $» \mathrm{D}$ a habt ihr gar nichts zu sagen zu mir, [...] dafür sind wir nicht draußen gewesen und haben im Graben gelegen, daß ihr hetzt« (94). 
Schrullen versteht man nicht, denn er sei schließlich, wird ihm vorgehalten, »in einer so ausgezeichneten Verfassung zurückgekommen «. ${ }^{27}$

Genau das aber ist das Problem: die Unsichtbarkeit und demzufolge Unkommunizierbarkeit mancher Kriegsfolgen; im Scbwierigen nimmt die daraus resultierende Diskrepanz erheiternde Formen an. Komisch ist hier, und zwar im vollsten Sinne des Wortes, dass und wie plötzlich ein Abstand, eine Erfahrungskluft in der Gesellschaft aufreißt, die um keinen Preis überbrückt, ja auch nur besprochen werden kann. »Nach einem unglücklichen Krieg müssen Komödien geschrieben werden«, bemerkt Hofmannsthal 1921 in dem Essay Die Ironie der Dinge. ${ }^{28}$ Die Komödie ist nicht nur ein effektvolles Gegengift zum heroischen oder tragischen Pathos der Kriegsverlautbarungen und ihrem sprichwörtlichen "Ernst der Lage«, sie bringt auf verträglich-heitere Weise auch die verstörenden Aspekte der Kriegserfahrung zu Bewusstsein und gewährt durch ihre Ironisierung einen comic relief. Bei Döblin geht es offenkundig nicht ganz so heiter zu, doch auch ihm gerät die Hervorkehrung evidenter Kriegsfolgen stellenweise zur Burleske, in der Hypochondrie oder Hochstapelei das Sagen haben. Der Vater eines vermeintlich falsch oder zu spät behandelten Kindes, das im Krankenhaus verstarb, erregt sich: »Ich bin ein Krüppel, wir haben im Feld geblutet, uns läßt man warten, mit uns kann man machen.« (115)

Kriegskrüppel, und solche, die es scheinen wollen, bilden durch den Roman eine Parade von derber und drastischer Komik. Da ist der schlimme Reinhold, der als Stotterer im Soldatenmantel eingeführt wird, und da ist die »Schicksalstragödie des Fliegers Beese-Arnim«, der einmal aus 1700 Metern wheruntergeschossen« wurde, so richtig aber erst nach dem Krieg abstürzt, Geld und Familienehre verliert und am Ende eine Prostituierte erschießt (303). Da sind jede Menge als Statisten auftretende Kriegsinvaliden, und da ist der durch einen Unfall gelähmte Johann Kirbach, der auf selbstfabriziertem Fahrgestell mit seinen Bildpostkarten auf Mitleidstournee geht und sein Schicksal mit dem »Ausbruch des Weltkrieges« (246) in ursächliche Verbindung bringt. Ein junger Bursche, den dieser Fall exemplarisch dünkt, redet sich in Rage:

27 Hugo von Hofmannsthal, »Der Schwierige«, in: ders., Dramen $I V$, Bernd Schoeller (Hrsg.), Frankfurt a.M. 1979, S. 337, 336.

28 Hugo von Hofmannsthal, »Die Ironie der Dinge« (1921), in: ders., Reden und Aufsätze, Bd. 2, Bernd Schoeller/Ingeborg Beyer-Ahlert (Hrsg.), Frankfurt a.M. 1979, S. 138-141, hier S. 138.
Und fängt nun im Lokal ein Geschrei darüber an und was sie auch mit seinem Vater gemacht haben, der hat einen Brustschuß, und jetzt hat er knappe Luft, aber mit einmal soll das bloß Nervenleiden sein, und die Rente haben sie ihm gekürzt, und nächstens kriegt er gar keine mehr. (246)

Unter der Last steigender Sozialausgaben werden die Folgen des Kriegs psychologisiert, individualisiert, bagatellisiert. Was einst im kollektiven Taumel der allgemeinen Mobilmachung begann, wird für die bewegungsunfähigen Krüppel plötzlich zu einer Frage der Selbstbeteiligung. Die Kneipenrunde allerdings ist sich uneins, je nach Grad der eigenen Betroffenheit:

'Puh! Die Krüppel - für die sollten sie überhaupt keinen Sechser geben. sSo siehste aus. Erst rausholen inn Krieg und dann nicht zahlen.o Gehört sich ooch so, Mensch. Wenn du woanders ne Dummheit machst, kriegste du ooch nich noch was druff gezahlt.< (247)

Widerfahrenes Schicksal oder selbstverschuldetes Unglück, da muss man sich gat nicht entscheiden, denn für Biberkopf kommt eins zum anderen. „Die Häuser, die rutschenden Dächer, ein hoher finsterer Hof, es braust ein Ruf wie Donnerhall, juvivallerallera, so hat es angefangen.« (225) Als er dies überlegt, sist er ein Krüppel«; wie in lapidarer Bilanz vermerkt wird: »Er hat einen Schlag auf den Kopf bekommen, man hat ihm einen Stoß vor die Brust gegeben, dann hat man ihn durch die Tür vor ein Auto geworfen. Das hat ihn überfahren. Sein Arm ist weg.« (226) Schon bald nach der Amputation wird Franz lernen, mit den Scherzen über seinen fehlenden Arm die Frauen zum Lachen zu bringen. Dieser negative Bildungsroman steht nicht an, den Verlust sogar als Fortschritt zu verbuchen. Denn zum verborgenen Trauma hat Franz jetzt die manifeste Verstümmelung und obendrein noch, gut sichtbar, "für besondere Feierlichkeiten" (254) ein Eisernes Kreuz, mit dem sein Leiden erstmals und vollständig als kriegsbedingt Anerkennung findet. Biberkopf ist, um hier die schöne Formulierung Ernst Blochs zweckentfremdet sarkastisch zur Geltung zu bringen, als einarmiges Kriegsopfer endlich zur Kenntlichkeit entstellt.

Denn wie im Falle des Scbwierigen lagen auch Biberkopfs Schwierigkeiten (und die der Gesellschaft mit ihm) hauptsächlich darin, Krieg und Trauma nicht in eine schlüssige Verbindung bringen zu können. Der Text wiederum evoziert diesen blinden Fleck dadurch, dass er die Analogie von Biberkopfs Großstadterfahrungen mit der traumatischen Zeit im Schützengraben zwar immer wieder suggeriert, aber eben nicht ausspricht. Zu den unterschwelligen Hinweisen gehört beispielsweise die Vierjahresfrist, die vom historischen ,Ereignis abgelöst ihr Eigenleben fühtt: »Vier Jahre nach achtzehn war ich in Berlin. Länger hat vorher der ganze Krieg nicht gedauert, stimmt 
doch.« (85) Die quantitative Koinzidenz beweist nichts und ist doch ein relevantes ästhetisches Merkmal. Der von Biberkopf gezogene Vergleich indes bleibt stumpf und offenbart seine Unfähigkeit, aus den im Krieg geopferten Jahren soziale Anerkennung zu ziehen:

$\mathrm{Na}$, und haben wir hier was von Arras gemerkt [...]? Haben gehabt Inflation, $\mathrm{Pa}$ $\mathrm{Na}$, und haben willionen, Billionen, kein Fleisch, keine Butter, schlimmer als vorher pierscheine, Millionen, Billionen, kein Fleisch, keine Butter, schlimmer ausrechnen das haben wir alles gemerkt, [...] und wo ist Arras gewesen, kannst du ausrechnen haben den Bauern Kartoffeln geklaut. (85)

Das ziellose Herumlaufen, dessen Motorik auch die sprunghafte Wahrnehmungsweise und den Erzählton bestimmt, charakterisiert einen depravierten Kriegsheimkehrer, der seiner Vorkriegsexistenz entfremdet ist und nirgends mehr hingehört. Mit dem rapiden Wandel der Verhältnisse, der in der Inflationszeit zusätzliche Dramatik erhielt, war den Kriegsteilnehmern der Boden unter den Füßen entzogen. Eine ähnliche Verbindung zwischen Inflation, Ortlosigkeit und Geschichtsverlust hat Walter Benjamin in seinem Erzäbler-Aufsatz 1936 gezogen. Die Entwertung und Enteignung von Erfahrungen, wie sie Benjamin an der deutschen Nachkriegszeit abliest, betrifft vor allem den Umgang mit den Kriegserlebnissen selbst, in denen Etfahrungsbildung als solche grundlegend erschüttert worden war:

Denn nie sind Erfahrungen gründlicher Lügen gestraft worden als die strategiDen durch den Stellungskrieg, die wirtschaftlichen durch die Inflation, die körschen durch den Stellungskrieg, die wirtschaftlichen durch die Inflation, ${ }^{29}$

Die Abwesenheit des Krieges im renormalisierten Wirtschafts- und Gesellschaftsleben macht es so schwierig, seiner traumatischen Spätfolgen habhaft zu werden. Gerade in dem Sinne ist der Krieg verloren, dass mit dem Wissen der Opfer auch seine Gewaltdimension für die Weimarer Republik abhanden gekommen ist: »Einen Krieg gewinnen oder verlieren, das greift, wenn wir der Sprache folgen, so tief in das Gefüge unseres Daseins ein, daß wir damit auf Lebenszeit an Malen, Bildern, Funden reicher oder ärmer geworden sind. $\ll^{30}$

Die Konsequenz dieses Gedankens liegt, aus heutiger Sicht zumindest, auf der Hand: Wer den Krieg in der von Benjamin gemeinten Weise wverlo-

29. Walter Benjamin, „Der Erzähler. Betrachtungen zum Werk Nikolai Lesskows«, in ders Gesammelte Scbriften, Bd. 2, Rolf Tiedemann/Hermann Schweppenhäuser (Hrsg.), S. 438-465, hier S. 439 .

Walter Benjamin, 》Theorien des deutschen Faschismus«, in: ders., Gesammelte Schriften, Bd. 3, Rolf Tiedemann/Hermann Schweppenhäuser (Hrsg.), S. 238-250, hier S. $242 \mathrm{f}$. ren« hat, der muss ihn wiederholen. Die politische Ästhetik des Döblin'schen Romans aber zielt darauf, ihn wiederzuholen, ins kulturelle Gedächtnis zurück. Berlin Alexanderplatz ist, als Großstadtroman, zugleich eine Schrift gegen die Abwesenheit des Krieges. Das betrifft nicht nur die Angstträume Biberkopfs, in deren Metaphernbestand ganze Panzerschlachten geschlagen werden:

Die Welt ist von Eisen, man kann nichts machen, sie kommt wie eine Walze an, auf einen zu, da ist nichts zu machen, da kommt sie, da läuft sie, da sitzen sie drin, das ist ein Tank, Teufel mit Hörnern und glühenden Augen drin, sie zerfleischen einen, sie sitzen da, mit ihren Ketten und Zähnen zerreißen sie einen. (210)

Wichtiger, und auch strukturell belastbar, ist Döblins Entdeckung des Krieges im Großstadtbetrieb selbst; in Gestalt eines Schlachtfeldes, auf dem das Töten nie aufgehört hat:

Die Eldenaer Straße entlang zichen sich die schmutziggrauen Mauern, oben mit Stacheldraht. [...] Gelbe Verwaltungsgebäude, ein Obelisk für Gefallene aus dem Weltkrieg. Und rechts und links langgestreckte Hallen mit gläsernen Dächern [...]. Die Rinderhalle, die Schweinehalle, die Schlachträume: Totengęrichte für die Tiere, schwingende Beile, du kommst mir nicht lebend raus. Friedliche Straßen grenzen an, Straßmannstraße, Liebigstraße, Proskauer, Gartenanlagen, in denen Leute spazieren. Sie wohnen warm beieinander, wenn einer erkrankt und Halsschmerzen hat, kommt der Arzt gelaufen. (136f.)

Inmitten der friedliebenden bürgerlichen Sekurität gibt es ein Areal, das nicht nur mit seinem stummen Kriegerdenkmal an den Weltkrieg erinnert. Es ist der ganz normale Schlachtbetrieb, der in seiner Logistik den Vergleich mit Mobilmachung und Truppentransporten, mit Materialschlacht, Schützengräben und Massensterben förmlich erzwingt, sieht man ihn mit den $\mathrm{Au}$ gen dieser Schilderung:

Aus den Provinzen rollt das Vieh ran, Exemplare der Gattung Schaf, Schwein, Rind, aus Ostpreußen, Pommern, Brandenburg, Westpreußen. Über die Viehrampen mähen, blöken sie herunter. Die Schweine grunzen und schnüffeln am Boden, sie sehen nicht, wo es hingeht, die Treiber mit den Stecken laufen hinterher. In die Ställe, da legen sie sich hin, liegen weiß, feist beieinander, schnarchen, schlafen. Sie sind lange getrieben worden, dann gerüttelt in den Wagen, jetzt vibriert nichts unter ihnen, nur kalt sind die Fliesen, sie wachen auf, drängen an andere. [...] In Furcht klettert eins über die Leiber der andern, das andere klettert hinterher, schnappt, die unten wühlen sich auf, die beiden plumpen herunter, suchen sich. (137)

Zuletzt, als das massenhafte Töten vollbracht ist, bleibt nurmehr das reflexhafte eigenmotorische Zucken der Körperteile - auch den Ärzten der Kriegsneurotiker wohlbekannt -, während von einem Ichbewusstsein, wie es die 
kantianische Rechtsphilosophie unterstellt, bei jedweder Art von Schlachtvieh nicht die Rede sein kann:

[...] das Beil ist heruntergesaust, getaucht in das Gedränge mit der stumpfen Seite [...] das Beil ist heruntergesaust, getauche in ...]. Das zappelt unten. Das strampelt. Das auf einen Kopf, noch einen Kopf. [...]. Das zappelt unten. Das strampechen die schleudert sich auf die Seite. Das weiß nichts mehr. Und liegt da. Was machen die Beine, der Kopf. Aber das macht das Schwein nicht, das machen die Beine als Privatperson. (139)

Mehr noch als die anderen Bilder, Stimmen und Rhythmen der Stadt findet (traße in Biberkopfs Angstvisionen einen Widerhall, denn er hat es selbst erlebt. Sein Delirium im Außenposten Berlin-Buch ist aus ästhetischer Sicht ein Zitat des Schlachthofs, symptomatologisch dagegen eine Wiederkehr des Schlachtfeldes:

Da blitzt ein Beil durch die Luft. [...] Es blitzt, es fällt, es fallbeilt im Halbbogen vorn vor durch die Luft, schlägt ein, schlägt ein, ein neues saust, ein neues saust, ein nochwing hoch, schlag nieder, hack ein. (431)

Der industrialisierte Tötungsakt, d.h. die massenhafte und mechanische Verwandlung von Lebewesen in tote Waren, gibt auch bei der psychiatrischen Zurichtung von Körper und Geist den Takt vor: »Es werden auf dem Block geschlagen von seinem Körper Stück um Stück. Sein Körper schiebt sich automatisch vor, muß sich vorschieben, er kann nicht anders.« (390) Diese Reprise des Schlachthofs in einer Anstalt mit dem sprechenden Ortsnamen »Berlin-Buch« erhebt die Zerstückelung des Menschen zu einem Signum der Großstadtkultur, ja der Moderne schlechthin. Üblicherweise läuft, was hier in krudester Form ausphantasiert wird, viel alltäglicher $a b$, etwa bei der Einführung automatisierter Produktionstechnologien. Denn durch die funktionale Zerlegung seiner Gliedmaßen und seines Aktionsrepertoires wird der Mensch unblutig den Zielen einer fordistischen Produktionsweise eingepasst. Womit wir bei einem anderen Kriegsschauplatz der zwanziger Jahre sind. Im Zuge der Maschinisierung von Arbeitsabläufen waren schematisierte Verhaltensweisen, die in psychologischer Hinsicht als Indizien reduzierter Geistestätigkeit zu gelten hatten, durchaus erwünscht. Die Montage-Technik, als innovative Qualität der Biberkopfgeschichte vielfach hervorgehoben, kam dort, wo sie industriell angewandt wurde, durchaus nicht »mit Kleister und Schere ${ }^{31}$ aus. Bekanntlich bestimmt das Fließband die arbeitsphysiologischen Standards der modern times,

31 So Titel eines Aufsatzes von Jürgen Stenzel, der sich mit Döblins Arbeitsweise beschäftigt ("Mit Kleister und Schere. Zur Handschrift von Berlin Alexanderplatz", in: Text und Kritik, 13/14: Alfred Döblin/1966, 21972, S.39-44). indem es die stumpfsinnige Wiederholung immergleicher Handgriffe verlangt.

Solcherart verlässliche Monotonie findet ihr romaninternes Sinnbild im dumpfen »Rumm rumm« der Dampframme am Alexanderplatz:

Rumm rumm wuchtet vor Aschinger auf dem Alex die Dampframme. [...] Rumm rumm haut die Dampframme auf dem Alexanderplatz. [...] Ein Mann oben zieht immer eine Kette, dann pafft es oben, und ratz hat die Stange eins auf den Kopf

[...] Viele Menschen haben Zeit und gucken sich an, wie die Ramme haut. (165)

Weil sie arbeitslos sind, können sie dem Schauspiel ihrer Ersetzung beiwohnen und bestaunen, wie im repetierten Zusammenprall von Metallgewicht und Eisenstange stellvertretend auch die Physiognomie des Menschen gezüchtigt wird: $\gg \mathrm{Da}$ stehen die Männer und Frauen und besonders die Jungens und freuen sich, wie das geschmiert geht: ratz kriegt die Stange eins auf den Kopf.« (165) Erst die brutale Vereinfachung komplexer Bewegungen sichert ihre abweichungsfreie und endlose Wiederholbarkeit. Sie rüstet den Menschen nach den Erfordernissen der Maschinenarbeit zu. Zwischen organischem Leib und maschinellen Standards vermittelt im Falle Biberkopfs vornehmlich die "Psychotechnik" des Marschierens, ${ }^{32}$ mit der das Subjekt, vermeintlich auf traditionelle Rituale des Exerzierens und der soldatischen Haltung zurückfallend, sich zur industriellen Disposition hält und damit in einen leibhaftigen Modernisierungseffekt verwandelt. Biberkopf marschiert, »mit festem Schritt, links rechts, links rechts, keine Müdigkeit vorschützen« (291f.), und unterstellt sich damit den elementaren Rhythmen mechanischer Impulse und metallischer Gebärden.

Wenn der eigene Körper als Zugriffsobjekt der Maschinengewalt erlebt wird, so lassen sich umgekehrt die Baustellen des technisierten Stadtraums als Operationen an einer schmerzempfindlichen Extension des kollektiven Leibes verstehen. Besonders der Alexanderplatz, mit den Ausschachtungsarbeiten für die U-Bahn, der Verbreiterung der S-Bahn-Viadukts und der Demolition ganzer Häuserzeilen zugunsten moderner Bauquader, kann als Zentralstelle der Amputation gelten. Selbst der Volksmund macht mit und beginnt vorsorglich schon den Namen des Platzes zu amputieren: „Wind gibt es massenhaft am Alex, an der Ecke von Tietz zieht es lausig. Es gibt Wind, der pustet zwischen die Häuser rein und auf die Baugruben.« (166) Der Hölderlin-Leser Döblin aber weiß: Wo Zerfall und Leere, da wächst das Nährende auch. »Aschinger hat ein großes Café und Restaurant. Wer keinen

32 Schäffner, Die Ordnung des Wabns, S. 338. 
Bauch hat, kann einen kriegen, wer einen hat, kann ihn beliebig vergröBern.« Indem er Platz schafft für Neues, entleibt der Stadtraum sich ständig selbst:

An der Ecke Landsberger Straße haben sie Friedrich Hahn, ehemals Kaufhaus, ausverkauft, leergemacht und werden es zu den Vätern versammeln. [...] Wo Jürgens war, das Papiergeschäft, haben sie das Haus abgerissen und dafür einen Bauzaun hingesetzt. [...] Ein Müllhaufen liegt vor uns. Von Erde bist du gekommen, zu Erde sollst du wieder werden, wir haben gebauet ein herrlich Haus, nun geht hier kein Mensch weder rein noch raus. (167)

Als Baron Haussmann in Paris brutale Schneisen durch den mittelalterlichen Stadtkern schlagen ließ, um dem rebellischen Volk die Errichtung von Barrikaden zu erschweren, inspirierte das den Dichter Baudelaire zu seiner Bestimmung der Moderne als stets transitorischen Augenblick. Auf halber Strecke zwischen Vergänglichkeit und Ewigkeit angesiedelt sah Baudelaire auch das Wesen der poetischen Allegorie. Döblin hatte seinen Roman zunächst mit der Haussmannisierung Berlins eröffnen wollen, dem Blick auf die Bauwut am Alexanderplatz und jene Ikone des anthropomorphen, des mythischen Berlin, die während der Niederschrift der neuen Zeit weichen musste: "Alles ist mit Brettern belegt. Die Berolina stand vor Tietz, eine Hand ausgestreckt, war ein kolossales Weib, die haben sie weggeschleppt. Vielleicht schmelzen sie sie ein und machen Medaillen draus.« (165) Nur konsequent wäre es, wenn der Umbau des Platzes die allegorische Schutzfigur in einen Rohstoff für Gedenk- oder Tapferkeitsmedaillen verwandeln würde. Wir wissen - es herrscht immer Krieg in den Städten: »So ist kaputt Rom, Babylon, Ninive, Hannibal, Cäsar, alles kaputt, oh, denkt daran.« (167)

Die Erdaushebungen erinnern nicht nur an Schützengräben, auch sie fordern ihre Opfer - jenes Pferd beispielsweise, das in der Brunnenstraße aus dem für die U-Bahnlinie angelegten Schacht befreit werden muss (241). »In den Boden rin, in die Erde rin, wo es finster ist« (21), dorthin fühlt sich auch der Kriegsneurotiker wie magisch gezogen, ohne diesem elementaren Schutzreflex nachzugeben:

Mit Genugtuung wanderte Biberkopf weiter. [...] Aber dann glitten seine Blicke im Ruck die Häuserfronten hoch, prüften die Häuserfronten, versicherten sich, $\mathrm{daB}$ sie stillstanden und sich nicht regten, trotzdem eigentlich so ein Haus viele Fenster hat und sich leicht vornüber beugen kann. Das kann auf die Dächer übergehen, die Dächer mit sich ziehen; sic können schwanken. Zu schwanken können sie anfangen, zu schaukeln, zu schütteln. Rutschen können die Dächer, wie Sand schräg herunter, wie ein Hut vom Kopf. Sind ja alle, ja alle schräg aufgestellt, die ganze Reihe lang. (131)
Sieht Franz Biberkopf Gespenster? Jedenfalls steht er damit nicht allein da »Warum ist alles so schlecht gebaut, daß bisweilen hohe Häuser einstürzen, ohne daß man einen äußeren Grund finden könnte.«, fragt der Protagonist einer kleinen Prosaskizze Franz Kafkas.

Ich klettere dann über die Schutthaufen und frage jeden, dem ich begegne: „Wie konnte das nur geschehn! In unserer Stadt - ein neues Haus - das ist heute schon das fünfte - bedenken Sie doch.« Da kann mir keiner antworten ${ }^{33}$

Die Begegnung von Mensch und Stadt ist ein Schock, und ihr Schauplatz ein Schlachtfeld. "Als ich mich zum Schreiben niedersetzte«, berichtet Kafka über die Entstehung der Erzählung Das Urteil, wwollte ich [...] einen Krieg beschreiben, ein junger Mann sollte aus seinem Fenster eine Menschenmenge über die Brücke herankommen sehn, dann aber drehte sich mir alles unter den Händen ${ }^{34}$ Dieser Krieg, der so schlecht sich erzählen lässt, wird nicht von ungefähr in die Großstadt verlegt, denn in ihm kommen jene Verwerfungen zum Tragen, die das Wahrnehmungsgefüge der Menschen von Grund auf in Frage stellen. Mit den Worten Walter Benjamins:

Eine Generation, die noch mit der Pferdebahn zur Schule gefahren war, stand unter freiem Himmel in einer Landschaft, in der nichts unverändert geblieben war als die Wolken und unter ihnen, in einem Kraftfeld zerstörender Ströme und Explosionen, der winzige, gebrechliche Menschenkörper. ${ }^{35}$

Für Franz Biberkopf aber liegt das gemeinsame Dritte von Stadt und Krieg im unverdrossenen Marschieren, immerfort, solange die Häuserfront halten wird und die Erde ihn nicht verschlingt: »Und Schritt gefaßt und rechts und links und rechts und links, marschieren, marschieren, wir ziehen in den Krieg, [...] dem einen gehts grade, dem andern gehts krumm, der eine bleibt stehen, der andere fällt um, der eine rennt weiter, der andere liegt stumm« (454f.).

33 Franz Kafka, „Gespräch mit dem Beter«, in: ders., Gesammelte Werke in 2mölf Bänden, Bd. 1, Hans-Gerd Koch (Hrsg.), Frankfurt a.M. 1994, S. 299-306, hier S. 305

Franz Kafka, Briefe an Felice und andere Korrespondenz aus der Verlobungszeit, Erich

Heller/Jürgen Born (Hrsg.), Frankfurt a.M. 1976, S. 394 (02.06.1913).
Benjamin, »Der Erzähler«, S. 439 . 